\title{
The Parseval identity for $q$-Sturm-Liouville problems with transmission conditions
}

Nida Palamut Koşar ${ }^{1 *}$ (D)

\section{"Correspondence:} npkosar@gmail.com

'Department of Mathematics and Science Education, Gaziantep University, Gaziantep, Turkey

\section{Springer}

\begin{abstract}
In the present study, we investigate the existence of spectral functions and obtain the Parseval identity and expansion formula in eigenfunctions for the singular q-Sturm-Liouville problem with transmission conditions.
\end{abstract}

MSC: 34L10; 39A13; 34L 40

Keywords: q-Sturm-Liouville operator; Parseval identity; Spectral function

\section{Introduction and background}

The development of applications of the $q$-calculus has been of great interest recently. It has an especially important role in the different areas of mathematics, such as orthogonal polynomials, mathematical physics, the calculus of variations, statistical mechanics, theory of various operators of convergence, quantum theory. More detailed concepts of the $q$-calculus can be obtained from books [1,2]. For a general introduction to the $q$-calculus, very useful studies have been done in [3-9].

It is well known that eigenfunction expansion problems are important for solving various problems, and there are lots of techniques for obtained (see $[3,10,11])$. On the other hand, many researchers have focused on certain generalizations of Sturm-Liouville problems. In particular, $q$-Sturm-Liouville eigenvalue problems were studied and a self-adjoint $q$-difference operator was formulated in a Hilbert space in [7, 12].

In [13] authors also studied the eigenfunction expansion for a certain $q$-Sturm-Liouville problem by using Titchmarch's technique and defined some concepts for deriving eigenfunction expansion problems.

Sturm-Liouville problems with transmission conditions have been investigated by many authors $[5,14,15]$ who gave asymptotic formulas for eigenvalues and the corresponding eigenfunction for these problems.

The existence of a spectral function for singular $q$-Sturm-Liouville operators on semiunbounded interval was proved in [4], and also they obtained the Parseval identity and on the expansion formula.

In this study, we consider a $q$-Sturm-Liouville expression as follows:

$$
\ell(y):=-\frac{1}{q} D_{q^{-1}} D_{q} y(\zeta)+u(\zeta) y(\zeta), \quad \zeta \in J:=[-1,0) \cup(0,1] .
$$

(c) The Author(s) 2021. This article is licensed under a Creative Commons Attribution 4.0 International License, which permits use, sharing, adaptation, distribution and reproduction in any medium or format, as long as you give appropriate credit to the original author(s) and the source, provide a link to the Creative Commons licence, and indicate if changes were made. The images or other third party material in this article are included in the article's Creative Commons licence, unless indicated otherwise in a credit line to the material. If material is not included in the article's Creative Commons licence and your intended use is not permitted by statutory regulation or exceeds the permitted use, you will need to obtain permission directly from the copyright holder. To view a copy of this licence, visit http://creativecommons.org/licenses/by/4.0/. 
Here, we denote $J_{1}:=[-1,0), J_{2}:=(0,1]$, and so $J:=J_{1} \cup J_{2}$. Suppose that the points -1 , 1 and 0 are $q$-regular for the differential expression $\ell . u$ is a real, Lebesque measurable function in $J$ and $u \in L_{q}^{1}\left(J_{k}\right), k=1,2$. Recall that 0 is a $q$-regular point of the function $u$ which belongs to $L_{q}^{1}[0-\varepsilon, 0+\varepsilon]$ for some $\varepsilon>0$.

The rest of the study is arranged as follows. In Sect. 2, we give some preliminaries for $q$-calculus. In Sect. 3, we investigate the existence of a spectral function. The Parseval identity and an expansion formula with eigenfunctions for a singular $q$-Sturm-Liouville problem with transmission conditions are obtained.

\section{Preliminaries}

We begin with some preliminary facts and notations for quantum calculus (see [2, 6]). Our main tools are $q$-derivative and $q$-integral. Let $q$ be any fixed constant with $q \in(0,1)$. We say that a set $A \subset \mathbb{R}$ is $q$-geometric if, for every $a \in A, q a \in A$. Also a $q$-difference equation is an equation which includes $q$-derivatives of a function defined on a $q$-geometric set $A$. The $q$-difference operator is denoted by $D_{q}$, the Jackson $q$-derivative of a function $\varphi: A \rightarrow$ $\mathbb{C}$ is defined by

$$
D_{q} \varphi(a)=\frac{\varphi(q a)-\varphi(a)}{q a-a}, \quad \forall a \in A \backslash\{0\} .
$$

We say that the point 0 in $A$ is the Jackson $q$-derivative at zero if the limit

$$
D_{q} \varphi(0)=\lim _{n \rightarrow \infty} \frac{\varphi\left(q^{n} a\right)-\varphi(0)}{q^{n} a} \quad(a \in A)
$$

exists and belongs to $\mathbb{C}$. Here, note that the value of the limit is independent of $a$ (see [8]). The Jackson $q$-integral is given by

$$
\int_{0}^{a} \varphi(\zeta) d_{q} \zeta=a(1-q) \sum_{k=0}^{\infty} q^{k} \varphi\left(q^{k} a\right) \quad(a \in A)
$$

where the series is convergent in [9]. Additionally, the following result is satisfied:

$$
\int_{a}^{b} \varphi(\zeta) d_{q} \zeta=\int_{0}^{b} \varphi(\zeta) d_{q} \zeta-\int_{0}^{a} \varphi(\zeta) d_{q} \zeta, \quad \forall a, b \in A
$$

The Jackson $q$-integration of $\varphi$ on $[0, \infty)$ is defined in [16] by the formula

$$
\int_{0}^{\infty} \varphi(\zeta) d_{q} \zeta=\sum_{n=-\infty}^{\infty} q^{n} \varphi\left(q^{n} a\right), \quad(a \in A)
$$

provided that some converges absolutely. A function $\varphi$ is $q$-regular at the point zero if the limit

$$
\lim _{n \rightarrow \infty} \varphi\left(a q^{n}\right)=\varphi(0), \quad(a \in A \cup\{0\})
$$

exists. Throughout the study, the functions will be assumed to be $q$-regular at zero. If $\varphi$ and $\psi$ are $q$-regular at zero, then the following equality holds:

$$
\int_{0}^{a} \psi(\zeta) D_{q} \varphi(\zeta) d_{q} \zeta+\int_{0}^{a} \varphi(q \zeta) D_{q} \psi(\zeta) d_{q} \zeta=\varphi(a) \psi(a)-\varphi(0) \psi(0) .
$$


The separable Hilbert space is $L_{q}^{2}(0, \infty):=\left\{\left.\varphi\left|\int_{0}^{\infty}\right| \varphi(\zeta)\right|^{2} d_{q} \zeta<\infty, \varphi:(0, \infty) \rightarrow \mathbb{C}\right\}$ with the norm

$$
\|\varphi\|:=\left(\int_{0}^{\infty}|\varphi(\zeta)|^{2} d_{q} \zeta\right)^{\frac{1}{2}}<\infty
$$

and given with the inner product

$$
\langle\varphi, \psi\rangle:=\int_{0}^{\infty} \varphi(\zeta) \overline{\psi(\zeta)} d_{q} \zeta, \quad \varphi, \psi \in L_{q}^{2}(0, \infty)
$$

(see [13]).

We call the $q$-Wronskian of $\varphi, \psi$ on $A$ if

$$
W_{q}[\varphi, \psi](a):=\varphi(a) D_{q} \psi(a)-\psi(a) D_{q} \varphi(a)
$$

exists.

\section{Main results}

In this section, we begin with the $q$-Sturm-Liouville equations as follows:

$$
\ell(y):=-\frac{1}{q} D_{q^{-1}} D_{q} y(\zeta)+u(\zeta) y(\zeta)=\lambda y, \quad \zeta \in J
$$

with the boundary conditions

$$
\begin{aligned}
& y(-1) \cos \alpha+D_{q^{-1}} y(-1) \sin \alpha=0, \\
& y(1) \cos \beta+D_{q^{-1}} y(1) \sin \beta=0
\end{aligned}
$$

and the transmission conditions

$$
\begin{aligned}
& y(0+)-\gamma_{1} y(0-)-\gamma_{2} D_{q} y(0-)=0, \\
& D_{q} y(0+)-\gamma_{3} y(0-)-\gamma_{4} D_{q} y(0-)=0,
\end{aligned}
$$

where $\lambda$ is a complex eigenparameter, the potential function $u \in L_{q}^{1}(J)$, and notice that it guarantees that $y(0 \mp)$ and $D_{q} y(0 \mp)$ in (3.4)-(3.5) make sense; here we assume that

$$
\gamma=\left|\begin{array}{ll}
\gamma_{1} & \gamma_{2} \\
\gamma_{3} & \gamma_{4}
\end{array}\right|>0
$$

Furthermore, the class $H_{q}=L_{q}^{2}\left(J_{1}\right) \oplus L_{q}^{2}\left(J_{2}\right)$ is introduced as a Hilbert space with the inner product

$$
\langle\varphi, \psi\rangle_{H_{q}}:=\int_{-1}^{0} \varphi_{1} \overline{\psi_{1}} d_{q} \zeta+\frac{1}{\gamma} \int_{0}^{1} \varphi_{2} \overline{\psi_{2}} d_{q} \zeta
$$

where

$$
\varphi(\zeta)=\left\{\begin{array}{ll}
\varphi_{1}(\zeta), \quad \zeta \in J_{1}, \\
\varphi_{2}\left(\zeta, \quad \zeta \in J_{2},\right.
\end{array} \quad \psi(\zeta)= \begin{cases}\psi_{1}(\zeta), & \zeta \in J_{1} \\
\psi_{2}(\zeta), & \zeta \in J_{2}\end{cases}\right.
$$


It can be easily obtained by direct manipulation from [17], (p.217). A compact resolvent of the regular self-adjoint boundary value problem (3.1)-(3.3), (3.4)-(3.5) with transmission was proved by authors in $[18,19]$, and they also showed that it has a completely discrete spectrum.

Let $\lambda_{m ; 1},(m \in N)$ denote the eigenvalues of this problem and by

$$
\begin{aligned}
& \phi_{m ; 1}(\zeta)= \begin{cases}\phi_{m ; 1}^{(1)}(\zeta), & \zeta \in J_{1}, \\
\phi_{m ; 1}^{(2)}(\zeta), & \zeta \in J_{2},\end{cases} \\
& \phi_{m ; 1}(\zeta):=\phi\left(\zeta, \lambda_{m ; 1}\right)
\end{aligned}
$$

the corresponding real-valued eigenfunctions which satisfy conditions (3.2)-(3.5).

If $\varphi \in H_{q}$ is a real-valued function with

$$
\varphi(\zeta)= \begin{cases}\varphi_{1}(\zeta), & \zeta \in J_{1} \\ \varphi_{2}(\zeta), & \zeta \in J_{2}\end{cases}
$$

then

$$
\begin{aligned}
\|\varphi\|_{H_{q}}^{2} & =\int_{-1}^{0}\left(\varphi_{1}(\zeta)\right)^{2} d_{q} \zeta+\frac{1}{\gamma} \int_{0}^{1}\left(\varphi_{2}(\zeta)\right)^{2} d_{q} \zeta \\
& =\sum_{m=1}^{\infty} \frac{1}{\alpha_{m ; 1}^{2}}\left[\int_{-1}^{0} \varphi_{1}(\zeta) \phi_{m ; 1}^{(1)}(\zeta) d_{q} \zeta+\frac{1}{\gamma} \int_{0}^{1} \varphi_{2}(\zeta) \phi_{m ; 1}^{(2)}(\zeta) d_{q} \zeta\right]^{2},
\end{aligned}
$$

where

$$
\alpha_{m ; 1}^{2}=\int_{-1}^{0}\left(\phi_{m ; 1}^{(1)}\right)^{2} d_{q} \zeta+\frac{1}{\gamma} \int_{0}^{1}\left(\phi_{m ; 1}^{(2)}\right)^{2} d_{q} \zeta
$$

is obtained. Here equality (3.7) is called the Parseval identity (see [5]).

Now we give a monotone increasing step function on $\mathbb{R}$ :

$$
\sigma_{1}(\lambda)= \begin{cases}-\sum_{\lambda<\lambda_{m ; 1}<0} \frac{1}{\alpha_{m ; 1}^{2}} & \text { for } \lambda<0, \\ \sum_{0 \leq \lambda_{m ; 1}<\lambda} \frac{1}{\alpha_{m ; 1}^{2}} & \text { for } \lambda \geq 0 .\end{cases}
$$

Thus, we can write (3.7) as

$$
\int_{-1}^{0}\left(\varphi_{1}(\zeta)\right)^{2} d_{q} \zeta+\frac{1}{\gamma} \int_{0}^{1}\left(\varphi_{2}(\zeta)\right)^{2} d_{q} \zeta=\int_{-\infty}^{+\infty} \Phi^{2}(\lambda) d \sigma_{1}(\lambda)
$$

where

$$
\Phi(\lambda)=\int_{-1}^{0} \varphi_{1}(\zeta) \phi_{m ; 1}^{(1)}(\zeta) d_{q} \zeta+\frac{1}{\gamma} \int_{0}^{1} \varphi_{2}(\zeta) \phi_{m ; 1}^{(2)}(\zeta) d_{q} \zeta
$$

We will obtain the Parseval identity for (3.1)-(3.5) from (3.9) by letting $1 \rightarrow \infty$. 
The function $\varphi$ is said to be of bounded variation on the interval $[a, b]$ if and only if there exists a positive constant $M$ such that

$$
\sum_{k=1}^{n}\left|\varphi\left(\zeta_{k}\right)-\varphi\left(\zeta_{k-1}\right)\right| \leq M
$$

for all finite partitions $\mathbb{P}=\left\{\zeta_{0}, \zeta_{1}, \ldots, \zeta_{n}\right\}$ of $[a, b]$.

If $\varphi:[a, b] \rightarrow \mathbb{R}$ is of bounded variation on $[a, b]$, then the total variation of $\varphi$ on $[a, b]$ is defined to be

$$
V_{a}^{b}(\varphi):=\sup \sum_{k=1}^{n}\left|\varphi\left(\zeta_{k}\right)-\varphi\left(\zeta_{k-1}\right)\right|,
$$

where the supremum is taken over all partitions of $[a, b]$ (see [5]).

Lemma 3.1 For arbitrary $M$, the formula

$$
V_{-M}^{M}\left(\sigma_{1}(\lambda)\right)=\sum_{-M<\lambda_{m ; 1}<M} \frac{1}{\alpha_{m ; 1}^{2}}=\sigma_{1}(M)-\sigma_{1}(-M)<\Upsilon
$$

holds for a positive constant $\Upsilon=\Upsilon(M)$.

Proof Assume first that $\sin \alpha \neq 0$. Since $\phi(\zeta, \lambda)$ is continuous on the domain $[-1,0] \mathrm{x}$ $[-M, M]$ with the condition $\phi(-1, \lambda)=\sin \alpha$, there exists a positive number $k$ such that

$$
\left(\frac{1}{k} \int_{-1}^{k} \phi_{m ; 1}^{(1)}(\zeta, \lambda) d_{q} \zeta\right)^{2}>\frac{\sin ^{2} \alpha}{2} .
$$

Let us define

$$
\varphi_{k}(\zeta):= \begin{cases}\frac{1}{k}, & -1 \leq \zeta<k \\ 0, & \zeta \geq k\end{cases}
$$

From (3.9) and (3.11), we get

$$
\begin{aligned}
\int_{-1}^{k} \varphi_{k}^{2}(\zeta) d_{q} \zeta & =\frac{k+1}{k^{2}} \\
& =\int_{-\infty}^{\infty}\left(\frac{1}{k} \int_{-1}^{k} \phi_{m ; 1}^{(1)}(\zeta, \lambda) d_{q} \zeta\right)^{2} d \sigma_{1}(\lambda) \\
& \geq \int_{-M}^{M}\left(\frac{1}{k} \int_{-1}^{k} \phi_{m ; 1}^{(1)}(\zeta, \lambda) d_{q} \zeta\right)^{2} d \sigma_{1}(\lambda) \\
& >\frac{1}{2} \sin ^{2} \alpha \int_{-M}^{M} d \sigma_{1}(\lambda) \\
& =\frac{1}{2} \sin ^{2} \alpha\left[\sigma_{1}(M)-\sigma_{1}(-M)\right],
\end{aligned}
$$

so it satisfies inequality (3.10). 
If $\sin \alpha=0$, we give a formula for the function $\varphi_{k}(\zeta)$ by

$$
\varphi_{k}(\zeta):= \begin{cases}\frac{1}{k^{2}}, & -1 \leq \zeta<k \\ 0, & \zeta \geq k\end{cases}
$$

Thus, we obtain (3.10) by applying the Parseval identity.

Let us now mention the following well-known Helly's first and second theorems in [20]. Firstly, recall Helly's first theorem that given a uniformly bounded sequence $\left\{\psi_{n}\right\}$ of monotone increasing real functions on $[a, b]$, there exists a subsequence $\left\{\psi_{n_{k}}\right\}$ of $\left\{\psi_{n}\right\}$ converging to a monotone increasing real function $\psi$ on $[a, b]$.

Secondly, given a sequence $\left\{\psi_{n}\right\}$ of monotone increasing real functions on $[a, b]$, converging to a monotone increasing real function $\psi$, for every continuous function $\varphi$ on $[a, b]$, we have

$$
\lim _{n \rightarrow \infty} \int_{a}^{b} \varphi(\lambda) d \psi_{n}(\lambda)=\int_{a}^{b} \varphi(\lambda) d \psi(\lambda) .
$$

We introduce the Hilbert space $H:=L_{q}^{2}\left(J_{1}\right) \oplus L_{q}^{2}\left(J_{3}\right),\left(J_{1}:=[-1,0), J_{3}:=(0, \infty)\right)$ with the inner product

$$
\langle\varphi, \psi\rangle_{H}:=\int_{-1}^{0} \varphi_{1} \overline{\psi_{1}} d_{q} \zeta+\frac{1}{\gamma} \int_{0}^{\infty} \varphi_{2} \overline{\psi_{2}} d_{q} \zeta
$$

where

$$
\varphi(\zeta)=\left\{\begin{array}{ll}
\varphi_{1}(\zeta), \quad \zeta \in J_{1}, \\
\varphi_{2}(\zeta), \quad \zeta \in J_{3},
\end{array} \quad \psi(t)= \begin{cases}\psi_{1}(\zeta), & \zeta \in J_{1} \\
\psi_{2}(\zeta), & \zeta \in J_{3}\end{cases}\right.
$$

We assume that $\sigma$ is any nondecreasing function for $-\infty<\lambda<\infty$. Let us define all measurable real functions of Hilbert space by $L_{\sigma}^{2}(\mathbb{R})$ which satisfies

$$
\int_{-\infty}^{\infty} \varphi^{2}(\lambda) d \sigma(\lambda)<\infty
$$

with the inner product

$$
\langle\varphi, \psi\rangle_{\sigma}:=\int_{-\infty}^{\infty} \varphi(\lambda) \psi(\lambda) d \sigma(\lambda)
$$

The fundamental result of the study is given as follows.

Theorem 3.2 The nondecreasing function $\sigma(\lambda)$ on $-\infty<\lambda<\infty$ for q-Sturm-Liouville problem (3.1)-(3.5) satisfies the following properties:

(i) If

$$
\varphi(\zeta)= \begin{cases}\varphi_{1}(\zeta), \quad \zeta \in J_{1} \\ \varphi_{2}(\zeta), \quad \zeta \in J_{3}\end{cases}
$$


is a real-valued function and $\varphi$ belongs to $H$, then there is a function $\Phi \in L_{\sigma}^{2}(\mathbb{R})$ such that

$$
\begin{array}{r}
\lim _{n \rightarrow \infty} \int_{-\infty}^{\infty}\left[\Phi(\lambda)-\int_{-1}^{0} \varphi_{1}(\zeta) \phi_{m ; 1}^{(1)}(\zeta, \lambda) d_{q} \zeta\right. \\
\left.-\frac{1}{\gamma} \int_{0}^{n} \varphi_{2}(\zeta) \phi_{m ; 1}^{(2)}(\zeta, \lambda) d_{q} \zeta\right]^{2} d \sigma(\lambda)=0
\end{array}
$$

and the Parseval identity

$$
\|\varphi\|_{H}^{2}=\int_{-1}^{0}\left(\varphi_{1}(\zeta)\right)^{2} d_{q} \zeta+\frac{1}{\gamma} \int_{0}^{\infty}\left(\varphi_{2}(\zeta)\right)^{2} d_{q} \zeta=\int_{-\infty}^{\infty} \Phi^{2}(\lambda) d \sigma(\lambda)
$$

(ii) The integral $\int_{-\infty}^{\infty} \Phi(\lambda) \phi(\zeta, \lambda) d \sigma(\lambda)$ converges to $\varphi$ in $H$; that is,

$$
\begin{aligned}
& \lim _{n \rightarrow \infty}\left[\int_{-1}^{0}\left(\varphi_{1}(\zeta)-\int_{-n}^{n} \Phi(\lambda) \phi_{m ; 1}^{(1)}(\zeta, \lambda) d \sigma(\lambda)\right)^{2} d_{q} \zeta\right. \\
& \left.+\frac{1}{\gamma} \int_{0}^{\infty}\left(\varphi_{2}(\zeta)-\int_{-n}^{n} \Phi(\lambda) \phi_{m ; 1}^{(2)}(\zeta, \lambda) d \sigma(\lambda)\right)^{2} d_{q} \zeta\right]=0
\end{aligned}
$$

It should be known that the function $\sigma$ is said to be a spectral function for boundary value problem (3.1)-(3.5).

Proof We may assume that

$$
\varphi_{\xi}(\zeta)= \begin{cases}\varphi_{1 ; \xi}(\zeta), & \zeta \in[-1,0) \\ \varphi_{2 ; \xi}(\zeta), & \zeta \in(0, \xi]\end{cases}
$$

satisfies three conditions as follows:

(a) Let $\varphi_{\xi}(\zeta)$ be identically zero outside the set $[-1,0) \cup(0, \xi]$ with $\xi<1$.

(b) Let $\varphi_{\xi}(\zeta)$ and $D_{q} \varphi_{\xi}(\zeta)$ be $q$-regular functions at zero.

(c) Let $\varphi_{\xi}(\zeta)$ satisfy boundary conditions (3.1)-(3.5).

Applying Parseval identity (3.9) to the function $\varphi_{\xi}(\zeta)$, we obtain

$$
\int_{-1}^{0}\left(\varphi_{1 ; \xi}(\zeta)\right)^{2} d_{q} \zeta+\frac{1}{\gamma} \int_{0}^{\xi}\left(\varphi_{2 ; \xi}(\zeta)\right)^{2} d_{q} \zeta=\int_{-\infty}^{\infty} \Phi_{\xi}^{2}(\lambda) d \sigma(\lambda)
$$

where

$$
\Phi_{\xi}(\lambda)=\int_{-1}^{0} \varphi_{1 ; \xi}(\zeta) \phi_{m ; 1}^{(1)}(\zeta, \lambda) d_{q} \zeta+\frac{1}{\gamma} \int_{0}^{\xi} \varphi_{2 ; \xi}(\zeta) \phi_{m ; 1}^{(2)}(\zeta, \lambda) d_{q} \zeta
$$

Because $\phi(\zeta, \lambda)$ satisfies (3.1), it is clear that

$$
\phi(\zeta, \lambda)=\frac{1}{\lambda}\left[-\frac{1}{q} D_{q^{-1}} D_{q} \phi(\zeta, \lambda)+u(\zeta) \phi(\zeta, \lambda)\right] .
$$


From (3.14) we get

$$
\begin{aligned}
\Phi_{\xi}(\lambda)= & \frac{1}{\lambda} \int_{-1}^{0} \varphi_{1 ; \xi}(\zeta)\left[-\frac{1}{q} D_{q^{-1}} D_{q} \phi_{m ; 1}^{(1)}(\zeta, \lambda)+u(\zeta) \phi_{m ; 1}^{(1)}(\zeta, \lambda)\right] d_{q} \zeta \\
& +\frac{1}{\gamma} \frac{1}{\lambda} \int_{0}^{\xi} \varphi_{2 ; \xi}(\zeta)\left[-\frac{1}{q} D_{q^{-1}} D_{q} \phi_{m ; 1}^{(2)}(\zeta, \lambda)+u(\zeta) \phi_{m ; 1}^{(2)}(\zeta, \lambda)\right] d_{q} \zeta
\end{aligned}
$$

Since $\varphi_{\xi}(\zeta)$ is identically zero in a neighborhood of the point 1 and both $\varphi_{\xi}(\zeta)$ and $\phi(\zeta, \lambda)$ satisfy boundary conditions (3.1)-(3.3), taking by $q$-integration by parts, we obtain

$$
\begin{aligned}
\Phi_{\xi}(\lambda)= & \frac{1}{\lambda} \int_{-1}^{0} \phi_{m ; 1}^{(1)}(\zeta, \lambda)\left[-\frac{1}{q} D_{q^{-1}} D_{q} \varphi_{1 ; \xi}(\zeta)+u(\zeta) \varphi_{1 ; \xi}(\zeta)\right] d_{q} \zeta \\
& +\frac{1}{\gamma} \frac{1}{\lambda} \int_{0}^{1} \phi_{m ; 1}^{(2)}(\zeta, \lambda)\left[-\frac{1}{q} D_{q^{-1}} D_{q} \varphi_{2 ; \xi}(\zeta)+u(\zeta) \varphi_{2 ; \xi}(\zeta)\right] d_{q} \zeta .
\end{aligned}
$$

For any finite $M>0$, from (3.9) we have

$$
\begin{aligned}
\int_{|\lambda|>M} \Phi_{\xi}^{2}(\lambda) d \sigma_{1}(\lambda) & \\
\leq & \frac{1}{M^{2}} \int_{|\lambda|>M}\left\{\int_{-1}^{0} \phi_{m ; 1}^{(1)}(\zeta, \lambda)\left[-\frac{1}{q} D_{q^{-1}} D_{q} \varphi_{1 ; \xi}(\zeta)+u(\zeta) \varphi_{1 ; \xi}(\zeta)\right] d_{q} \zeta\right. \\
& \left.+\frac{1}{\gamma} \int_{0}^{1} \phi_{m ; 1}^{(2)}(\zeta, \lambda)\left[-\frac{1}{q} D_{q^{-1}} D_{q} \varphi_{2 ; \xi}(\zeta)+u(\zeta) \varphi_{2 ; \xi}(\zeta)\right] d_{q} \zeta\right\}^{2} d \sigma_{1}(\lambda) \\
\leq & \frac{1}{M^{2}} \int_{-\infty}^{+\infty}\left\{\int_{-1}^{0} \phi_{m ; 1}^{(1)}(\zeta, \lambda)\left[-\frac{1}{q} D_{q^{-1}} D_{q} \varphi_{1 ; \xi}(\zeta)+u(\zeta) \varphi_{1 ; \xi}(\zeta)\right] d_{q} \zeta\right. \\
& \left.+\frac{1}{\gamma} \int_{0}^{1} \phi_{m ; 1}^{(2)}(\zeta, \lambda)\left[-\frac{1}{q} D_{q^{-1}} D_{q} \varphi_{2 ; \xi}(\zeta)+u(\zeta) \varphi_{2 ; \xi}(\zeta)\right] d_{q} \zeta\right\}^{2} d \sigma_{1}(\lambda) \\
= & \frac{1}{M^{2}} \int_{-1}^{0}\left[-\frac{1}{q} D_{q^{-1}} D_{q} \varphi_{1 ; \xi}(\zeta)+u(\zeta) \varphi_{1 ; \xi}(\zeta)\right]^{2} d_{q} \zeta \\
& +\frac{1}{M^{2}} \frac{1}{\gamma} \int_{0}^{\xi}\left[-\frac{1}{q} D_{q^{-1}} D_{q} \varphi_{2 ; \xi}(\zeta)+u(\zeta) \varphi_{2 ; \xi}(\zeta)\right]^{2} d_{q} \zeta .
\end{aligned}
$$

From (3.14), we obtain that

$$
\begin{aligned}
& \left|\int_{-1}^{0}\left(\varphi_{1 ; \xi}(\zeta)\right)^{2} d_{q} \zeta+\frac{1}{\gamma} \int_{0}^{\xi}\left(\varphi_{2 ; \xi}(\zeta)\right)^{2} d_{q} \zeta-\int_{-M}^{M} \Phi_{\xi}^{2}(\lambda) d \sigma_{1}(\lambda)\right| \\
& <\frac{1}{M^{2}} \int_{-1}^{0}\left[-\frac{1}{q} D_{q^{-1}} D_{q} \varphi_{1 ; \xi}(\zeta)+u(\zeta) \varphi_{1 ; \xi}(\zeta)\right]^{2} d_{q} \zeta \\
& \quad+\frac{1}{M^{2}} \frac{1}{\gamma} \int_{0}^{\xi}\left[-\frac{1}{q} D_{q^{-1}} D_{q} \varphi_{2 ; \xi}(\zeta)+u(\zeta) \varphi_{2 ; \xi}(\zeta)\right]^{2} d_{q} \zeta .
\end{aligned}
$$

We know that the set $\left\{\sigma_{1}(\lambda)\right\}$ is bounded from Lemma 3.1. A sequence $\left\{\psi_{k}\right\}\left(\psi_{k} \rightarrow \infty\right)$ such that the function $\sigma_{1 ; \psi_{k}}(\lambda)$ converges to a monotone function $\sigma(\lambda)$ can be found from 
Helly's first and second theorems. By taking limit with respect to $\left\{\psi_{k}\right\}$ in (3.16), we get

$$
\begin{aligned}
& \left|\int_{-1}^{0}\left(\varphi_{1 ; \xi}(\zeta)\right)^{2} d_{q} \zeta+\frac{1}{\gamma} \int_{0}^{\xi}\left(\varphi_{2 ; \xi}(\zeta)\right)^{2} d_{q} \zeta-\int_{-M}^{M} \Phi_{\xi}^{2}(\lambda) d \sigma(\lambda)\right| \\
& <\frac{1}{M^{2}} \int_{-1}^{0}\left[-\frac{1}{q} D_{q^{-1}} \varphi_{1 ; \xi}(\zeta)+u(\zeta) \varphi_{1 ; \xi}(\zeta)\right]^{2} d_{q} \zeta \\
& \quad+\frac{1}{M^{2}} \frac{1}{\gamma} \int_{0}^{\xi}\left[-\frac{1}{q} D_{q^{-1}} \varphi_{2 ; \xi}(\zeta)+u(\zeta) \varphi_{2 ; \xi}(\zeta)\right]^{2} d_{q} \zeta
\end{aligned}
$$

Therefore, letting $M \rightarrow \infty$, we get

$$
\int_{-1}^{0}\left(\varphi_{1 ; \xi}(\zeta)\right)^{2} d_{q} \zeta+\frac{1}{\gamma} \int_{0}^{\xi}\left(\varphi_{2 ; \xi}(\zeta)\right)^{2} d_{q} \zeta=\int_{-\infty}^{+\infty} \Phi_{\xi}^{2}(\lambda) d \sigma(\lambda)
$$

Let $\varphi$ be any real function on $H$. It is known that there is a sequence of functions $\left\{\varphi_{\xi}(\zeta)\right\}$ satisfying conditions (3.1)-(3.5) and such that

$$
\lim _{\xi \rightarrow \infty}\left[\int_{-1}^{0}\left(\varphi_{1}(\zeta)-\varphi_{1 ; \xi}(\zeta)\right)^{2} d_{q} \zeta+\frac{1}{\gamma} \int_{0}^{\xi}\left(\varphi_{2}(\zeta)-\varphi_{2 ; \xi}(\zeta)\right)^{2} d_{q} \zeta\right]=0
$$

Let

$$
\Phi_{\xi}(\lambda)=\int_{-1}^{\infty} \varphi_{1 ; \xi}(\zeta) \phi_{m ; 1}^{(1)}(\zeta, \lambda) d_{q} \zeta+\frac{1}{\gamma} \int_{-1}^{\infty} \varphi_{2 ; \xi}(\zeta) \phi_{m ; 1}^{(2)}(\zeta, \lambda) d_{q} \zeta
$$

Then from this we can get

$$
\int_{-1}^{0}\left(\varphi_{1 ; \xi}(\zeta)\right)^{2} d_{q} \zeta+\frac{1}{\gamma} \int_{0}^{\infty}\left(\varphi_{2 ; \xi}(\zeta)\right)^{2} d_{q} \zeta=\int_{-\infty}^{+\infty} \Phi_{\xi}^{2}(\lambda) d \sigma(\lambda)
$$

Since

$$
\int_{-1}^{0}\left(\varphi_{1 ; \xi_{1}}(\zeta)-\varphi_{1 ; \xi_{2}}(\zeta)\right)^{2} d_{q} \zeta+\frac{1}{\gamma} \int_{0}^{\infty}\left(\varphi_{2 ; \xi_{1}}(\zeta)-\varphi_{2 ; \xi_{2}}(\zeta)\right)^{2} d_{q} \zeta \rightarrow 0
$$

as $\xi_{1}, \xi_{2} \rightarrow \infty$, we have

$$
\int_{-\infty}^{+\infty}\left(\Phi_{\xi_{1}}(\lambda)-\Phi_{\xi_{2}}(\lambda)\right)^{2} d \sigma(\lambda) \rightarrow 0
$$

as $\xi_{1}, \xi_{2} \rightarrow \infty$. Accordingly there is a limit function $\Phi$ such that

$$
\int_{-1}^{0}\left(\varphi_{1}(\zeta)\right)^{2} d_{q} \zeta+\frac{1}{\gamma} \int_{0}^{\infty}\left(\varphi_{2}(\zeta)\right)^{2} d_{q} \zeta=\int_{-\infty}^{+\infty} \Phi^{2}(\lambda) d \sigma(\lambda)
$$

holds by the completeness of the space $L_{\sigma}^{2}(\mathbb{R})$.

Our next aim is to see that

$$
K_{\xi}(\lambda)=\int_{-1}^{0} \varphi_{1}(\zeta) \phi_{m ; 1}^{(1)}(\zeta, \lambda) d_{q} \zeta+\frac{1}{\gamma} \int_{0}^{\xi} \varphi_{2}(\zeta) \phi_{m ; 1}^{(2)}(\zeta, \lambda) d_{q} \zeta
$$


converges to $\Phi$ as $\xi \rightarrow \infty$ in the space $L_{\sigma}^{2}(\mathbb{R})$. Let $\psi$ be another real-valued function in $H$. By the same way, let $\Psi(\lambda)$ be defined by $\psi$. It is clear that for

$$
\begin{aligned}
& \psi(\zeta):= \begin{cases}\psi_{1}(\zeta), & \zeta \in[-1,0) \cup[0, \xi] \\
\psi_{2}(\zeta), & \zeta \in(\xi, \infty)\end{cases} \\
& \int_{-1}^{0}\left(\varphi_{1}(\zeta)-\psi_{1}(\zeta)\right)^{2} d_{q} \zeta+\frac{1}{\gamma} \int_{0}^{\infty}\left(\varphi_{2}(\zeta)-\psi_{2}(\zeta)\right)^{2} d_{q} \zeta=\int_{-\infty}^{+\infty}[\Phi(\lambda)-\Psi(\lambda)]^{2} d \sigma(\lambda)
\end{aligned}
$$

Let us define

$$
\psi(\zeta)= \begin{cases}\varphi(\zeta), & \zeta \in[-1,0) \cup[0, \xi] \\ 0, & \zeta \in(\xi, \infty)\end{cases}
$$

then

$$
\int_{-\infty}^{+\infty}\left[\Phi(\lambda)-K_{\xi}(\lambda)\right]^{2} d \sigma(\lambda)=\frac{1}{\gamma} \int_{\xi}^{\infty}\left(\varphi_{2}(\zeta)\right)^{2} d_{q} \zeta \rightarrow 0,
$$

as $\xi \rightarrow \infty$, which proves that $K_{\xi}$ converges to $\Phi$ in $L_{\sigma}^{2}(\mathbb{R})$ as $\xi \rightarrow \infty$. This gives us (i).

Now let us give the proof of (ii). Assume that the real functions $\varphi, \psi \in H$ and $\Phi(\lambda), \Psi(\lambda)$ are their Fourier transforms respectively (see [10]). Then $\Phi \mp \Psi$ are transforms of $\varphi \mp \psi$. Consequently, by (3.13) we have

$$
\begin{aligned}
& \int_{-1}^{0}\left[\varphi_{1}(\zeta)+\psi_{1}(\zeta)\right]^{2} d_{q} \zeta+\frac{1}{\gamma} \int_{0}^{\infty}\left[\varphi_{2}(\zeta)+\psi_{2}(\zeta)\right]^{2} d_{q} \zeta=\int_{-\infty}^{+\infty}[\Phi(\lambda)+\Psi(\lambda)]^{2} d \sigma(\lambda) \\
& \int_{-1}^{0}\left[\varphi_{1}(\zeta)-\psi_{1}(\zeta)\right]^{2} d_{q} \zeta+\frac{1}{\gamma} \int_{0}^{\infty}\left[\varphi_{2}(\zeta)-\psi_{2}(\zeta)\right]^{2} d_{q} \zeta=\int_{-\infty}^{+\infty}[\Phi(\lambda)-\Psi(\lambda)]^{2} d \sigma(\lambda) .
\end{aligned}
$$

Let us subtract the resulting two equations side by side, we have

$$
\int_{-1}^{0} \varphi_{1}(\zeta) \psi_{1}(\zeta) d_{q} \zeta+\frac{1}{\gamma} \int_{0}^{\infty} \varphi_{2}(\zeta) \psi_{2}(\zeta) d_{q} \zeta=\int_{-\infty}^{+\infty} \Phi(\lambda) \Psi(\lambda) d \sigma(\lambda)
$$

which may be called the generalized Parseval identity. Set

$$
\varphi_{\tau}^{(j)}(\zeta)=\int_{-\tau}^{+\tau} \Phi(\lambda) \phi_{m ; 1}^{(j)}(\zeta, \lambda) d \sigma(\lambda), \quad j=1,2,
$$

where $\Phi$ is the function defined in (3.12). Let $\psi \in H$ be a real function which equals zero outside the set $[-1,0) \cup(0, \mu]$. Thus we obtain

$$
\begin{gathered}
\int_{-1}^{0} \varphi_{\tau}^{(1)}(\zeta) \psi_{1}(\zeta) d_{q} \zeta+\frac{1}{\gamma} \int_{0}^{\mu} \varphi_{\tau}^{(2)}(\zeta) \psi_{2}(\zeta) d_{q} \zeta \\
=\int_{-1}^{0}\left[\int_{-\tau}^{\tau} \Phi(\lambda) \phi_{m ; 1}^{(1)}(\zeta, \lambda) d \sigma(\lambda)\right] \psi_{1}(\zeta) d_{q} \zeta \\
\quad+\frac{1}{\gamma} \int_{0}^{\mu}\left[\int_{-\tau}^{\tau} \Phi(\lambda) \phi_{m ; 1}^{(2)}(\zeta, \lambda) d \sigma(\lambda)\right] \psi_{2}(\zeta) d_{q} \zeta
\end{gathered}
$$




$$
\begin{aligned}
& =\int_{-\tau}^{\tau} \Phi(\lambda)\left[\int_{-1}^{0} \phi_{m ; 1}^{(1)}(\zeta, \lambda) \psi_{1}(\zeta) d_{q} \zeta+\frac{1}{\gamma} \int_{0}^{\mu} \phi_{m ; 1}^{(2)}(\zeta, \lambda) \psi_{2}(\zeta) d_{q} \zeta\right] d \sigma(\lambda) \\
& =\int_{-\tau}^{\tau} \Phi(\lambda) \Psi(\lambda) d \sigma(\lambda) .
\end{aligned}
$$

Subtracting (3.17) and (3.18), we have

$$
\begin{aligned}
& \int_{-1}^{0}\left(\varphi_{1}(\zeta)-\varphi_{\tau}^{(1)}(\zeta)\right) \psi_{1}(\zeta) d_{q} \zeta+\frac{1}{\gamma} \int_{0}^{\infty}\left(\varphi_{2}(\zeta)-\varphi_{\tau}^{(2)}(\zeta)\right) \psi_{2}(\zeta) d_{q} \zeta \\
& \quad=\int_{|\lambda|>\tau} \Phi(\lambda) \Psi(\lambda) d \sigma(\lambda) .
\end{aligned}
$$

Using the Cauchy-Schwarz inequality, we obtain

$$
\begin{aligned}
& \left(\int_{-1}^{0}\left(\varphi_{1}(\zeta)-\varphi_{\tau}^{(1)}(\zeta)\right) \psi_{1}(\zeta) d_{q} \zeta+\frac{1}{\gamma} \int_{0}^{\infty}\left(\varphi_{2}(\zeta)-\varphi_{\tau}^{(2)}(\zeta)\right) \psi_{2}(\zeta) d_{q} \zeta\right)^{2} \\
& \quad \leq \int_{|\lambda|>\tau} \Phi^{2}(\lambda) d \sigma(\lambda) \int_{|\lambda|>\tau} \Psi^{2}(\lambda) d \sigma(\lambda)
\end{aligned}
$$

If we carry out this inequality to the function

$$
\psi(\zeta)= \begin{cases}\varphi_{\tau}(\zeta)-\varphi(\zeta), & \zeta \in[-1,0) \cup(0, \mu], \\ 0, & \zeta \in(\mu, \infty),\end{cases}
$$

we get

$$
\begin{aligned}
& \int_{-1}^{0}\left(\varphi_{1}(\zeta)-\varphi_{\tau}^{(1)}(\zeta)\right)^{2} d_{q} \zeta+\frac{1}{\gamma} \int_{0}^{\infty}\left(\varphi_{2}(\zeta)-\varphi_{\tau}^{(2)}(\zeta)\right)^{2} d_{q} \zeta \\
& \quad \leq \int_{|\lambda|>\tau} \Phi^{2}(\lambda) d \sigma(\lambda) .
\end{aligned}
$$

Since the above inequality is not dependent on $\mu$, the result is achieved by letting $\tau \rightarrow$ $\infty$.

\section{Conclusion}

In this study, we investigate the existence of a spectral function for the singular $q$-SturmLiouville problem with transmission conditions on a closed interval. We prove the Parseval identity with the help of the inner product in class $H_{q}=L_{q}^{2}\left(J_{1}\right) \oplus L_{q}^{2}\left(J_{2}\right)$ as Hilbert space. We also give the expansion formula in the eigenfunctions.

Acknowledgements

Not applicable.

Funding

Not applicable.

Abbreviations

Not applicable.

Availability of data and materials

Not applicable. 
Competing interests

The author declares that she has no competing interests.

Authors' contributions

The author carried out the research of this paper, and she read and approved the final version of the manuscript.

\section{Publisher's Note}

Springer Nature remains neutral with regard to jurisdictional claims in published maps and institutional affiliations.

Received: 12 February 2021 Accepted: 3 May 2021 Published online: 13 May 2021

\section{References}

1. Ernst, T.: The History of q-calculus and a New Method (2000) ISSN 1101-3591

2. Kac, V., Cheung, P.: Quantum Calculus. Springer, Berlin (2002)

3. Allahverdiev, B.P., Tuna, H.: An expansion theorem for q-Sturm-Liouville operators on the whole line. Turk. J. Math. 42(3), 1060-1071 (2018)

4. Allahverdiev, B.P., Tuna, H.: Eigenfunction expansion in the singular case for q-Sturm-Liouville operators. Casp. J. Math. Sci. 8(2), 91-102 (2019)

5. Allahverdiev, B.P., Tuna, H.: Eigenfunction expansion for singular Sturm-Liouville problems with transmission conditions. Electron. J. Differ. Equ. 2019, 03 (2019)

6. Annaby, M.H., Mansour, Z.S.: q-Fractional Calculus and Equations. Lecture Notes in Mathematics, vol. 2056 (2012)

7. Annaby, M.H., Mansour, Z.S.: Basic Sturm-Liouville problems. J. Phys. A, Math. Gen. 38, 3775-3797 (2005)

8. Jackson, F.H.: q-Difference equations. Am. J. Math. 32, 305-314 (1910)

9. Jackson, F.H.: On q-definite integrals. Pure Appl. Math. 41, 193-203 (1910)

10. Levitan, B.M., Sargsjan, I.S.: Sturm-Liouville and Dirac Operators. Mathematics and its Applications. Kluwer Academic, Dordrecht (1991)

11. Titchmarsh, E.C.: Eigenfunction Expansions Associated with Second-Order Differential Equations. Part I, 2nd edn. Clarendon Press, Oxford (1962)

12. Annaby, M.H., Mansour, Z.S.: Asymptotic formulae for eigenvalues and eigenfunctions of $q$-Sturm-Liouville problems. Math. Nachr. 284(4), 443-470 (2011)

13. Annaby, M.H., Mansour, Z.S., Soliman, I.A.: q-Titchmarsh-Weyl theory: series expansion. Nagoya Math. J. 205, 67-118 (2012)

14. Mukhtarov, O.Sh., Tunç, E.: Eigenvalue problems for Sturm Liouville equations with transmission conditions. Isr. J. Math. 2(144), 367-380 (2004)

15. Mukhtarov, O.Sh., Yakubov, S.: Problems for differential equations with transmission conditions. Appl. Anal. 81 1033-1064 (2002)

16. Hahn, W.: Beitrage zur Theorie der Heineschen Reiben. Math. Nachr. 2, 340-379 (1949) (in German)

17. Annaby, M.H.: q-Type sampling theorems. Results Math. 44, 214-225 (2013)

18. Dehghani, I., Akbarfam, A.J.: Resolvent operator and self-adjointness of Sturm-Liouville operators with a finite number of transmission conditions. Mediterr. J. Math. 11, 447-462 (2014)

19. Wang, A., Sun, J., Hao, X., Yao, S.: Completeness of eigenfunctions of Sturm-Liouville problems with transmission conditions. Math. Appl. Anal. 16, 299-312 (2009)

20. Kolmogorov, A.N., Fomin, S.V.: Introductory Real Analysis. Dover, New York (1970)

\section{Submit your manuscript to a SpringerOpen ${ }^{\circ}$ journal and benefit from:}

- Convenient online submission

- Rigorous peer review

- Open access: articles freely available online

- High visibility within the field

Retaining the copyright to your article

Submit your next manuscript at $>$ springeropen.com 